\title{
CARTILAGE DAMAGE INVOLVING EXTRUSION OF MINERALISABLE MATRIX FROM THE ARTICULAR CALCIFIED CARTILAGE AND SUBCHONDRAL BONE
}

\author{
A. Boyde ${ }^{1, *}$, C.M. Riggs ${ }^{2}$, A.J. Bushby ${ }^{3}$, B.McDermott ${ }^{4}$, G.L. Pinchbeck ${ }^{4}$ and P.D. Clegg $^{4}$ \\ ${ }^{1}$ Biophysics OGD, Barts and The London School of Medicine and Dentistry, Queen Mary University of London, \\ London E1 2AD, UK \\ ${ }^{2}$ Department of Veterinary Clinical Services, Hong Kong Jockey Club, Sha Tin Racecourse, Hong Kong, SAR China \\ ${ }^{3}$ Centre for Materials Research, Queen Mary University, London E1 4NS, UK \\ ${ }^{4}$ Dept. of Musculoskeletal Biology, Faculty of Health and Life Sciences, University of Liverpool, Leahurst, Neston \\ CH64 7TE, UK
}

\begin{abstract}
Arthropathy of the distal articular surfaces of the third metacarpal (Mc3) and metatarsal (Mt3) bones in the Thoroughbred racehorse $(\mathrm{Tb})$ is a natural model of repetitive overload arthrosis. We describe a novel pathology that affects the articular calcified cartilage (ACC) and subchondral bone (SCB) and which is associated with hyaline articular cartilage degeneration.

Parasagittal slices cut from the palmar quadrant of the distal condyles of the left Mc3/Mt3 of 39 trained Tbs euthanased for welfare reasons were imaged by point projection microradiography, and backscattered electron (BSE) scanning electron microscopy (SEM), light microscopy, and confocal scanning light microscopy. Mechanical properties were studied by nanoindentation. Data on the horses' training and racing career were also collected.

Highly mineralised projections were observed extending from cracks in the ACC mineralising front into the hyaline articular cartilage (HAC) up to two-thirds the thickness of the HAC, and were associated with focal HAC surface fibrillation directly overlying their site. Nanoindentation identified this extruded matrix to be stiffer than any other mineralised phase in the specimen by a factor of two. The presence of projections was associated with a higher cartilage Mankin histology score $(P<0.02)$ and increased amounts of gross cartilage loss pathologically on the condyle $(P<0.02)$. Presence of projections was not significantly associated with: total number of racing seasons, age of horse, amount of earnings, number of days in training, total distance galloped in career, or presence of wear lines.
\end{abstract}

Key words: Cartilage, bone, osteoarthritis, tidemark, microscopy, nanoindentation.

*Address for correspondence:

A. Boyde

Biophysics, Oral Growth and Development

Queen Mary University of London

New Road, London E1 2AD, U.K.

Telephone Number: +44 7957425992

E-mail: a.boyde@qmul.ac.uk

\section{Introduction}

Osteoarthritis (OA) is a multifactorial disease, and repetitive overload is a major candidate risk factor for osteoarthritis (OA). Mechanisms linking mechanical trauma and OA are poorly characterised. The distal third metacarpal (Mc3) and metatarsal (Mt3) bones in the Thoroughbred racehorse $(\mathrm{Tb})$ suffer from a range of osteochondral pathologies; examples include loss of articular cartilage (erosion, ulceration), formation of wear lines and subchondral bone fracture (Norrdin and Stover, 2006; Barr et al., 2009; Firth et al., 2009). Such pathologies can provide information about mechanisms relating to overload arthrosis in general.

Whilst a number of earlier studies in several species have identified alterations relating to osteoarthrosis in the osteochondral junctional region, few identify pathologies in that region in athletic animals. Earlier studies in the horse have shown a tendency for articular calcified cartilage (ACC) to crack more or less perpendicular to its mineralising front, parallel to the fibres forming the main component of the cartilage collagen (Boyde et al., 1999). In addition, cracks within ACC and subchondral bone (SCB) become repaired by the intercalation of a matrix which becomes densely mineralised (Boyde, 2003; Boyde and Firth, 2008). This infill material has been shown to extend up to, and to include, the level of the mineralisation front in ACC, and even slightly beyond (Boyde, 2003).

We had the opportunity to investigate changes in MC3/ Mt3 distal condyles from trained $\mathrm{Tb}$ racehorses destroyed for welfare reasons. Such material could be obtained from joints affected by a wide range of pathologies, immediately post-mortem, which allowed detailed investigation of early and late stages of a form of overload arthrosis. We aimed to determine the relationship between specific alterations in the osteochondral junction and the overlying hyaline articular cartilage (HAC), and relate such changes to the work intensity the horse had experienced during its racing and training career.

\section{Materials and Methods}

Material was examined from 39 Thoroughbred racehorses that were in, or had been retired from, active race training at the Hong Kong Jockey Club and were euthanased for welfare reasons. At post-mortem, the condylar articular pathology was scored; specifically relating to the presence of gross articular cartilage erosions or the presence of wear 
lines (Barr et al. 2009). From the left fore medial condyle and left hind lateral condyle of $\mathrm{Mc} 3 / \mathrm{Mt} 3,5$ parasagittal slices were cut from the palmar/plantar quadrant, some of which were fixed for 24 hours in $10 \%$ neutral formaldehyde solution prior to storage in $70 \%$ ethanol and then glycerol for transport. One slice (a-most abaxial) was macerated in $4 \%$ Tergazyme $^{\mathrm{TM}}$ (alkaline pronase enzyme detergent, Alconox Inc., New York, NY, USA); washed, dried, carbon coated and studied by 3D backscattered electron (BSE) imaging in the scanning electron microscope (SEM) to characterise the morphology of the mineralised front region (current tidemark of the articular cartilage). A separate slice (b) was embedded in poly(methyl methacrylate) (PMMA) and a block surface prepared by grinding and diamond polishing, and studied using quantitative BSE imaging (qBSE) in the SEM (Zeiss DSM962; Zeiss UK Ltd, Welwyn Garden City, Herts, UK) combined with autofluorescence confocal scanning light microscopy (CSLM) (Leica SP2; Leica Microsystems Ltd, Knowlhill, Milton Keynes, UK) for soft tissue histology (Boyde et al., 2005). Mechanical properties of the cut surface of the distal condyle in these blocks were studied by nanoindentation (UMIS-2000, CSIRO, Lindfield, NSW, Australia) (Ferguson et al., 2003). A further slice (e-most axial) was used for conventional histology following ethylenediaminetetraacetic acid (EDTA) decalcification, paraffin embedding, and staining with both haematoxylin and eosin, and safranin-O, and chondral pathology scored using a modified Mankin Score (McIlwraith et al., 2010). A further slice (d) was used in a separate study for molecular biological analysis of gene expression (data not shown here). A 'c' slice was used as a ground section for optical microscopy. All samples (with the exception of the first $12 \mathrm{~d}$ slices processed) were imaged with $26 \mathrm{kV}$ point projection X-ray microradiography (Faxitron; QADOS, Sandhurst, Berkshire, UK). As the findings here were part of a much larger study, we collected data relating to the horses' training and racing history (total number of racing seasons, age of horse, amount of earnings, number of days in training, total distance galloped in career) from management databases at the Hong Kong Jockey Club. Statistical analysis was performed using mixed effect regression models (S-Plus: Insightful, Chapel Hill, NC, USA) to allow for clustering within horse, as two condyles were assessed per animal. The presence of projections was associated with both gross pathological and histopathological scores, as well as the variables relating to the horses racing career. In all cases, $P<0.05$ denotes a significant difference.

\section{Results}

This study confirms the occasional presence of a very dense in-fill material, located in cracks in both ACC and SCB, which had been described in previous studies (Boyde, 2003; Boyde and Firth, 2008). Here, however, BSE SEM showed highly mineralised projections extending from cracks in the ACC mineralising front and for variable distances into the HAC in 8 of the 78 condyles, all from separate horses (Figs. 1-3). These mineralised projections could extend up to two-thirds through the thickness of the HAC (Fig 1c, 2a, $3 \mathrm{c})$. The range of mineralisation densities matches those seen in crack infill material in ACC and underlying SCB (Figs. 1a, 1g, 2c, 3a, 3b). The smallest projections tended to have a more uniform mineralisation density (Fig 3a), whereas more extensive projections contained domains with varying electron backscattering properties, indicative of varying mineral densities (Figs. 1a-c, 2c).

CSLM of the identical tissue volumes studied by BSE SEM demonstrated that the projections were surrounded by HAC which in some specimens appeared relatively normal on either side of the projection, with it being apparently displaced to provide the space (Figs. 1b-c, 2a-c). However, at other sites the surrounding cartilage matrix appeared abnormal and relatively acellular (Fig. 2d). No matter the size of the projection, they were associated with focal HAC fibrillation or loss directly overlying their site (Figs. $1 \mathrm{c}, 2 \mathrm{a}, 3 \mathrm{c}, 3 \mathrm{~d})$. In some cases, mineralised fragments were identified in the fibrillated superficial cartilage overlying the projections (Figs. 2c, 3b). At least in one case, several features could be seen in the sequence of plane-parallel parasagittal sections along the same mediolateral transect, a distance of $>8 \mathrm{~mm}$. In other cases they could be shown to be separate features in both the lateromedial and dorsopalmar/plantar planes (Fig. 3e).

We re-examined the PMMA embedded slices by both 3D optical microscopy and point projection digital microradiography. This approach showed examples of clustering of features within the thickness of a thick slice similar to those projections exposed at the cut surface (Figs. 1d-e).

Using conventional paraffin embedded decalcified and stained histology, we were frequently unable to identify the presence of these projections, and their site appeared as an artefactual full thickness split in the HAC. However, we were able to demonstrate regions with similar morphology by conventional histology in one specimen, their being best demonstrated with haematoxylin/eosin (H\&E) staining where there was very sparse detectable residual organic matrix (Fig. 2d).

The nanoindentation study showed the mineralised material in the extrusions to be stiffer by a factor of two than any other normal bone or cartilage phase in the specimen, including cement line matrix (Fig. 1f, 1g).

The presence of projections was associated with a greater cartilage Modified Mankin histology score $(P<0.02)$ (McIlwraith et al., 2010) and an increased pathological assessment of gross cartilage loss on the condyle $(P<0.02)$. The presence of projections was not significantly associated with any measure of longevity in racing, or work intensity - specifically; total number of racing seasons, age of horse, amount of earnings, number of days in training, total distance galloped in career - or the presence of wear lines (Table 1).

\section{Discussion}

The calcified projections from the mineralising front of ACC were discovered in this study by a combination of BSE and CSLM examination of plastic embedded block 

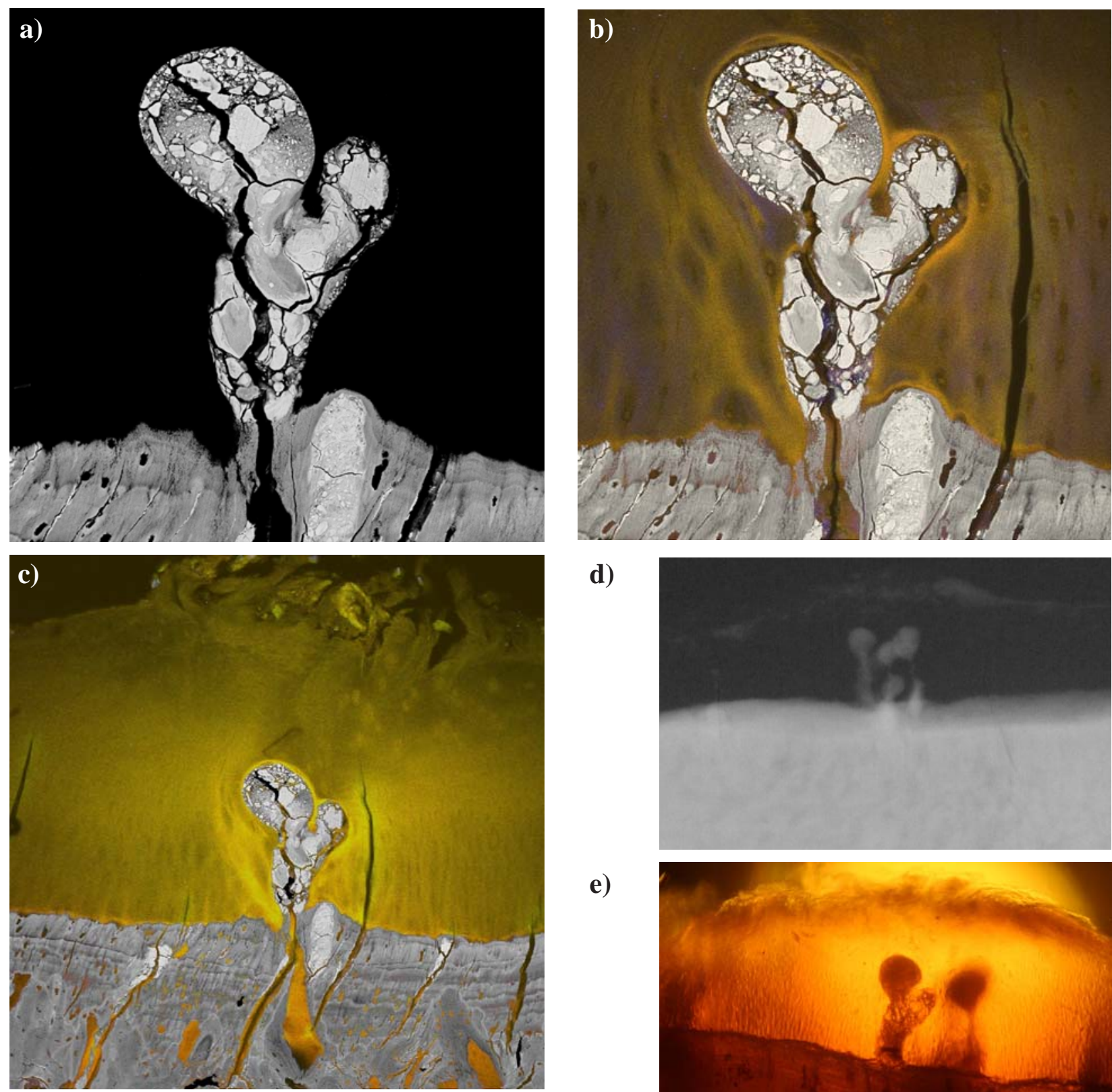

d)

e)
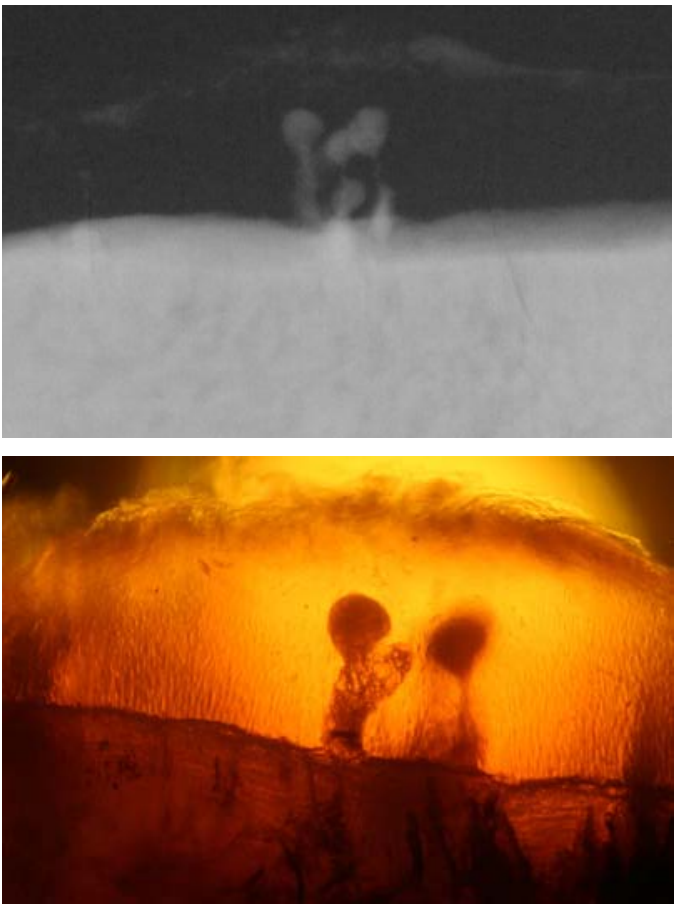

f)

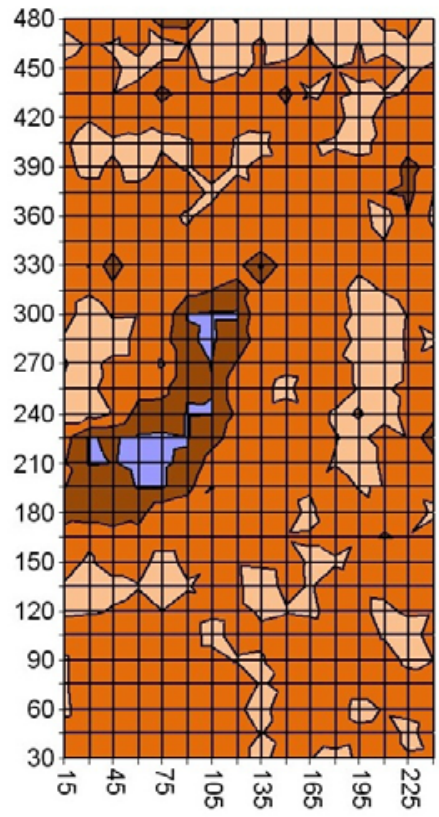

口30-35 ㄴ25-30 口20-25 ㅁ15-20 ㅁ10-15 口 5-10 ㅁ 0-5

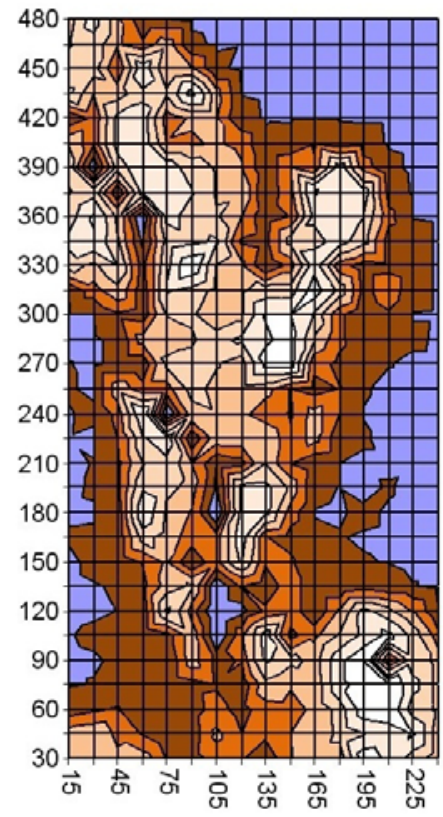


g)

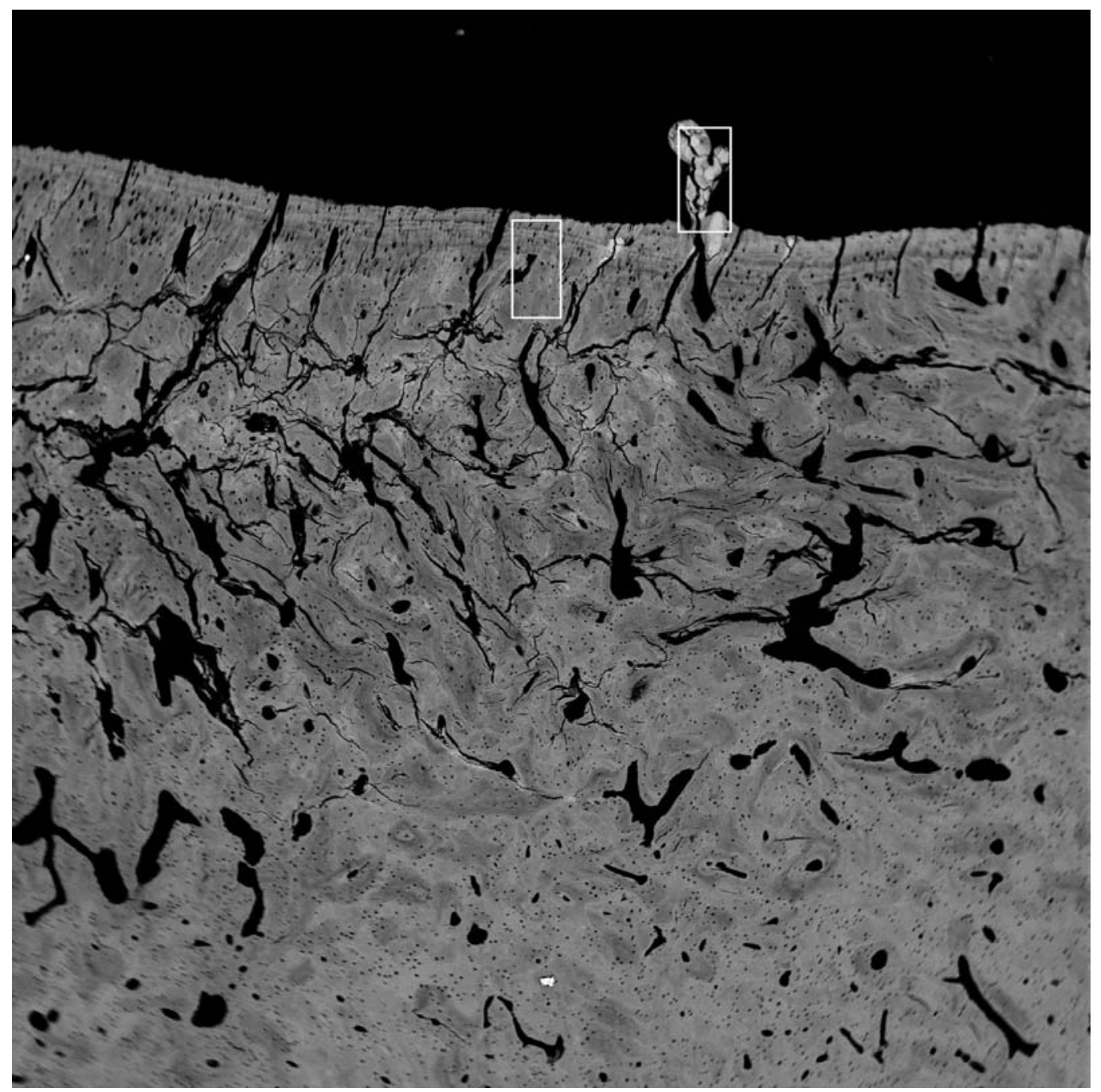

Fig. 1. Horse 5 in Table 1, neutered male, age 8 years, raced 56 times, modified Mankin score 18 (where range is 0-21). Medial palmar condyle of left Mc3. (a) $20 \mathrm{kV}$ backscattered electron image of polished PMMA embedded parasagittal ' $b$ ' slice. Grey levels reflect mineralisation density. Calcified protusion extends from the mineralising front of the ACC. Extensive black areas are hyaline articular cartilage, which gives zero BSE signal under these conditions. Cracks are an artefact of super-drying of the PMMA embedded tissue in the SEM vacuum. The thin layers of dense tissue within the fine cracks are not artefactual. There is a low profile protusion where a crack in ACC has been infilled to the right of the major projection extending into HAC. Field width and height $=614 \mu \mathrm{m}$; (b) $20 \mathrm{kV}$ BSE image shown in Fig. 1a (grey tones) combined with confocal green+red autofluorescence (yellows) and reflection (feint blue) showing that the calcified protusion extending from the mineralising front of the ACC is surrounded by relatively normal deeper hyaline articular cartilage (HAC). Field width and height $=614 \mu \mathrm{m}$; (c) Wider field showing combined $20 \mathrm{kV}$ BSE image (grey tones) combined with CSLM green+red autofluorescence (yellows) showing that the calcified protusion extends roughly half way through the thickness of the non-mineralised articular cartilage (HAC). The outer layers of the cartilage contain fewer chondrocytes and the surface layers (top) are fibrillated. Small fragments of mineralised tissue can be seen in the surface layer. Field width and height $=$ $1536 \mu \mathrm{m}$; (d) Same region in $35 \mathrm{kV}$ faxitron point projection $\mathrm{x}$-ray image at 6.6X projective magnification showing cluster of mineralised protusions within non-mineralised cartilage. Slice thickness $750 \mu \mathrm{m}$. Field width $=2933$ $\mu \mathrm{m}$. (e) Transmitted light image of region shown in Figs.1a etc., and as in Fig. 1d showing that there is more than one protusion from the ACC MF in this region. This is one side of a stereo-pair view obtained with the Edge 3D microscope. It clearly shows the fibrillated nature of the superficial cartilage layers (top). Slice thickness $750 \mu \mathrm{m}$, but depth of field much less than this. Dark shadows in lower part of image are vascular canals in bone. Field width $=2150 \mu \mathrm{m}$. (f) Nanoindentation studies were performed at a grid of points separated by $15 \mu \mathrm{m}$, as indicated by the grid intersections in this figure. Control region in left panel. Projection shown in Fig. 1a etc. is recognised in right panel. Colours represent indentation elastic modulus in GPa binned into ranges indicated in centre panel. The higher stiffness values found in patches in the centre of the protrusion exceed those found in normal ACC and bone in this and other samples. For exact areas of indentation data acquisition see Fig. 1g; (g) Wide field BSE SEM image with white boxes showing the areas from which the nanoindentation data shown in Fig 1f were obtained. There are large number of cracks in the subchondral bone indicating substantial damage via loads transmitted through HAC to SCB Field width $=4050 \mu \mathrm{m}$. 

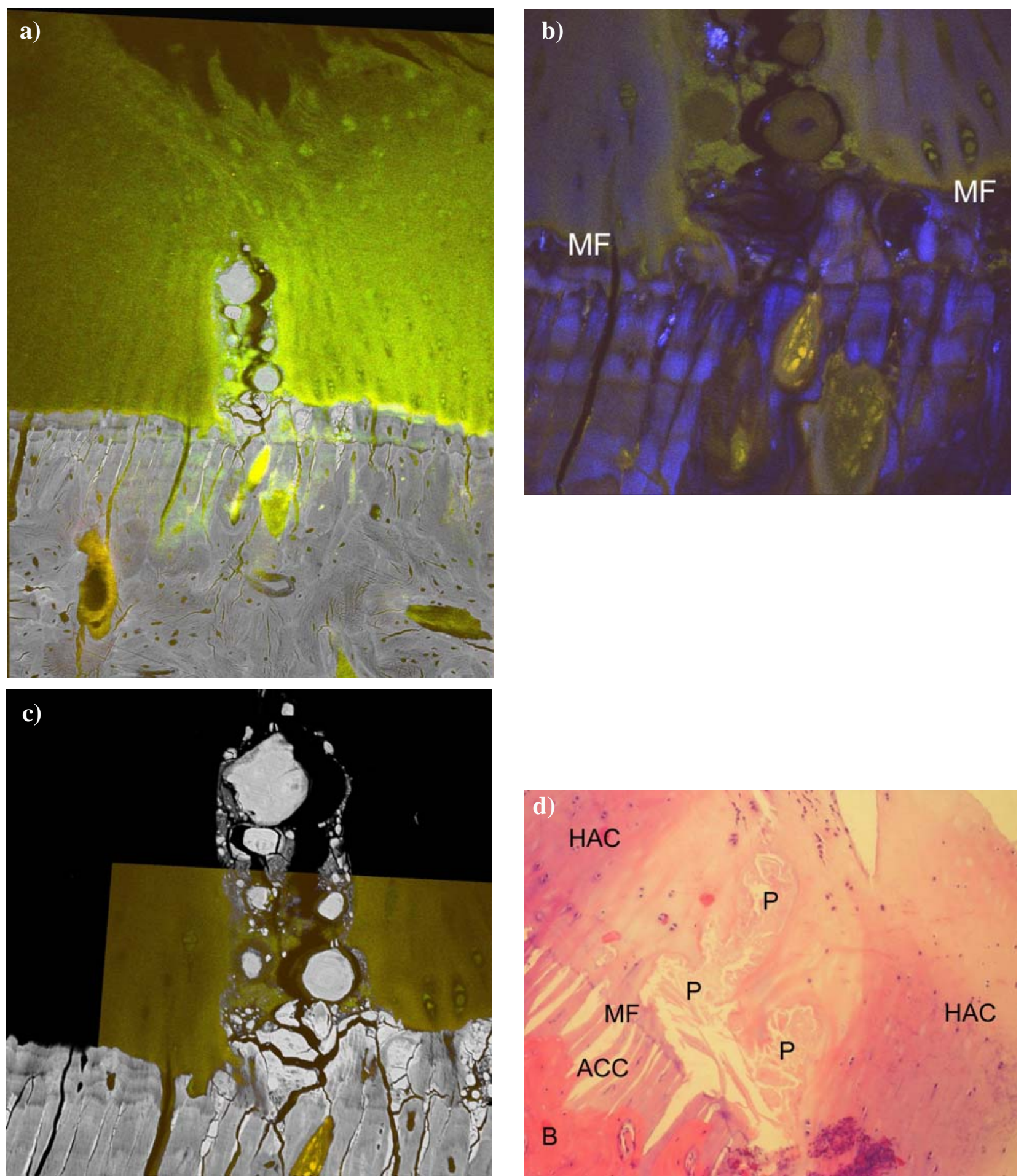

Fig. 2. Horse 1 in Table 1, neutered male, age 8 years, raced 49 times, modified Mankin score 8. Medial palmar condyle of left Mc3. (a) Combined $20 \mathrm{kV}$ backscattered electron and confocal autofluorescence green + red images of polished PMMA embedded parasagittal ' $b$ ' slice through. Calcified protusion extends from the mineralising front of the ACC and roughly halfway through HAC which is superficially fibrillated and eroded. Field width $=900 \mu \mathrm{m}$; (b) Confocal autofluorescence green+red and reflected light (blue) image of base of same protrusion. MF = 'tidemark' mineralising front of ACC. Morphology can be studied in the combined reflection and autofluorescence image, but the mineral content cannot be read without the BSE SEM image information. Field width $=384 \mu \mathrm{m}$; (c) Combined 20 $\mathrm{kV}$ backscattered electron and confocal autofluorescence green + red images of same calcified protrusion. Numerous cracks in all tissues are post preparation shrinkage-induced artefact: those lined with hyperdense material are real ante-mortem features. Field width $=450 \mu \mathrm{m}$; (d) Haematoxylin and eosin stained section of decalcified 'e' slice from same condyle shown in Figs. $2 \mathrm{a}-\mathrm{c} . \mathrm{B}=$ subchondral bone. ACC $=$ articular calcified cartilage with vertical artefactual splits. $\mathrm{MF}=$ 'tidemark' mineralising front. $\mathrm{HAC}=$ hyaline articular cartilage. $\mathrm{P}=$ 'protrusion'. Field width $=920 \mu \mathrm{m}$. 

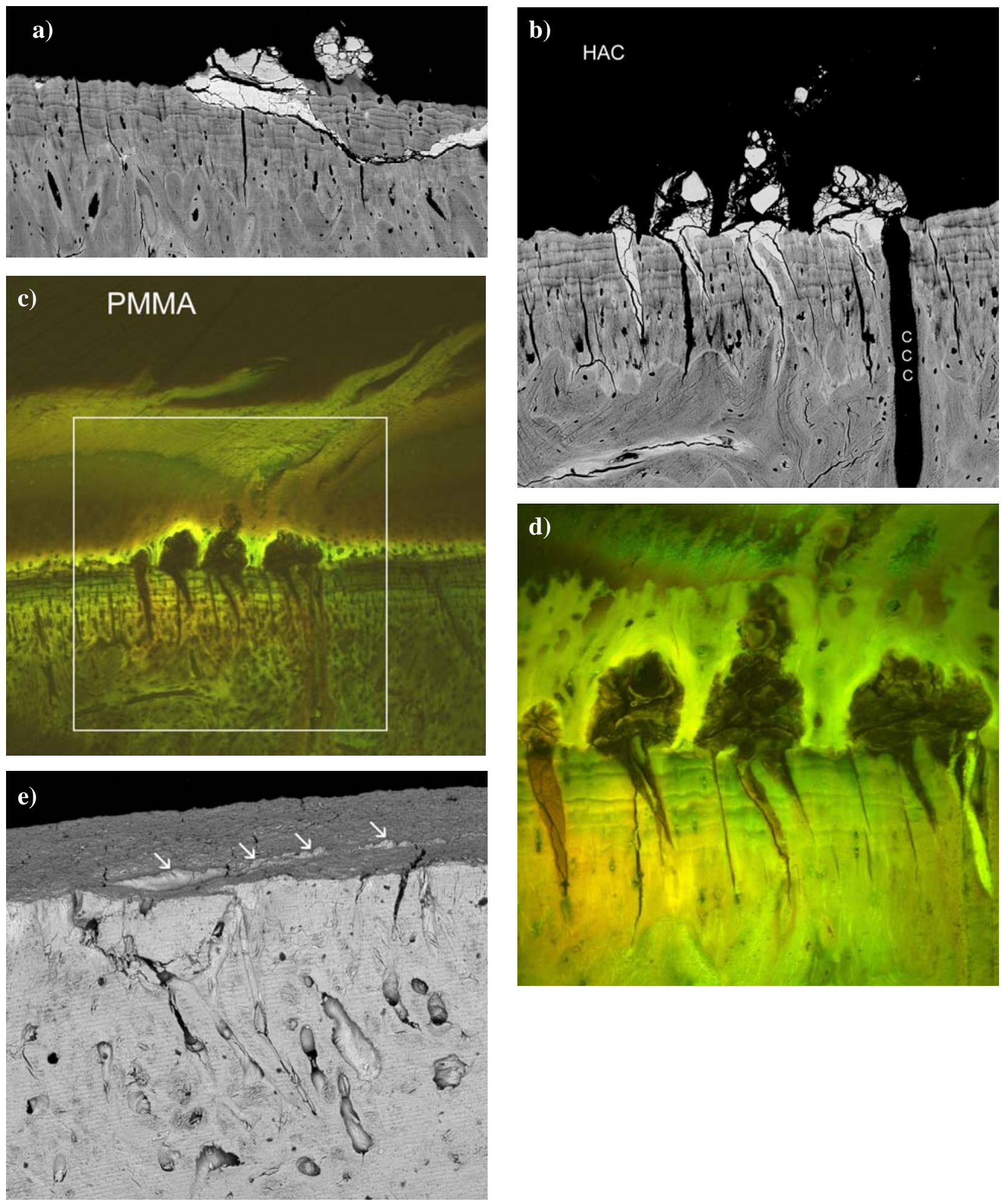

Fig. 3. Horse 4 in Table 1, neutered male, age 6 years, raced 24 times, modified Mankin score 9. Polished PMMA embedded parasagittal 'b' slice through medial palmar condyle of left Mc3. (a) $20 \mathrm{kV}$ BSE image taken at first polishing level [this sample was polished back $\sim 200 \mu \mathrm{m}$ prior to recording other parts of this figure]. Note two low profile protusions, one obviously connected to oblique tangential crack in ACC filled with dense material, the other outside the profile of the ACC MF. Field width = $1246 \mu \mathrm{m}$; (b) $20 \mathrm{kV}$ BSE image showing calcified protusions extending from the mineralising front of the ACC connected with dense infill in cracks in the ACC. Black areas at top are hyaline articular cartilage (HAC) which gives zero BSE signal under these conditions. $\mathrm{CCC}=$ a straight cutting cone canal. Field width $=1000 \mu \mathrm{m}$; (c) Confocal autofluorescence image of same region. White box shows area covered in BSE image. Note fibrillated and eroded HAC. PMMA = polished PMMA surface outside the fibrillated HAC. Field width $=1536 \mu \mathrm{m}$; (d) Higher magnification confocal autofluorescence image of same features. Image reconstructed from brightest fluorescence at each pixel from a through focus set covering a range of focus of $90 \mu \mathrm{m}$. Field width $=614$ $\mu \mathrm{m}$; (e) Macerated slice A. Arrows point to a line of protrusions of dense mineralised material from the mineralising front of the ACC following the direction of a parasagittal crack Field width $=2700 \mu \mathrm{m}$. 
Table 1. Pathological and racing career data relating to the eight horses in which projections were identified. POD score and cartilage loss on condyles were scored using the scoring system described in Barr et al. (2009).

$\begin{array}{ccccccccc}\begin{array}{c}\text { Horse } \\ \text { No. }\end{array} & \begin{array}{c}\text { Leg } \\ \text { (Left) }\end{array} & \begin{array}{c}\text { Retired } \\ \text { age }\end{array} & \begin{array}{c}\text { Carnings } \\ \text { earnis }\end{array} & \begin{array}{c}\text { Total } \\ \text { metres } \\ \text { raced }\end{array} & \begin{array}{c}\text { Total } \\ \text { days } \\ \text { training }\end{array} & \begin{array}{c}\text { Modified } \\ \text { Mankin } \\ \text { score } \\ \mathbf{( 0 - 2 1 )}\end{array} & \begin{array}{c}\text { POD } \\ \text { score } \\ (\mathbf{0 - 3 )}\end{array} & \begin{array}{c}\text { Cartilage } \\ \text { loss on } \\ \text { condyle } \\ \mathbf{( 0 - 3 )}\end{array} \\ 2 & \text { Fore } & 8 & 2347450 & 54100 & 2351 & 8 & 1 & 2 \\ 3 & \text { Fore } & 9 & 2211325 & 86600 & 2278 & 5 & 2 & 1 \\ 4 & \text { Hind } & 7 & 1175100 & 43600 & 1440 & 12 & 2 & 1 \\ 5 & \text { Fore } & 6 & 553700 & 29000 & 963 & 2 & 0 & 2 \\ 6 & \text { Fore } & 8 & 2966900 & 85200 & 1617 & 18 & 2 & 2 \\ 7 & \text { Find } & 6 & 1496000 & 44550 & 903 & 11 & 3 & 1 \\ 8 & \text { Fore } & 5 & 1093600 & 19600 & 1233 & 11 & 1 & 2 \\ & & 5 & 1844850 & 49050 & 1452 & 14 & 1 & 1\end{array}$

faces. Using conventional microscopic techniques of decalcification and paraffin embedding, we did not identify such features during the first rounds of our study. After we had initially discovered such features using BSE SEM and CSLM, we could demonstrate their presence using a variety of techniques. Detecting the projections by faxitron microradiography was facilitated retrospectively by altering contrast, brightness and window levels to see these slender features within the HAC zone. Equally, they could be seen in transmitted light microscopy in thick undecalcified glycerine cleared slices. These latter two methods have the advantage of simplicity and could be productively used in any survey prospecting for them, for example in studies in human traumatic OA. These features were frequently missed using conventional paraffin embedded decalcified and stained histology due to complete loss of the highly mineralised matrix, resulting in an apparently artefactual full thickness split in the HAC. Such splits have in the past been attributed by the authors to a sectioning artefact. In one specimen, a weakly staining matrix was identified using such microscopy techniques, and thus with appropriate technique such features could be identifiable using conventional histological approaches.

We hypothesise that the dense projections we describe in this paper arise by mineralisation of an aqueous, organic matrix, which fills ruptures in deep HAC and underlying ACC and SCB. Mineral fills water space within mineralised tissues. The most densely mineralised mammalian tissue is dental enamel, in which the protein matrix is degraded during mineralisation to permit the extremely high volume occupancy by mineral. The quantifiable BSE method applied to the study of soft tissue calcification found similar mineralisation densities, lying below those of enamel but above those of bone and ACC (Eidelman et al., 2009: see this paper for bibliography of soft tissue calcification). As with enamel, we presume that the organic matrix in these features is dispersed with conventional decalcification procedures, thus accounting for the fact that they have not been previously described. Not only would these features be substantially removed by demineralisation, but we speculate that they would not survive microtomy of intact undemineralised tissue since they would be ripped out of the block surface. Certainly any matrix is tenuous, since otherwise the very high levels of mineralisation could not be attained. We have not yet characterised the nature of the matrix of these features. HAC is a stiff rubbery material, which is able to transmit sudden impact loads sufficient to fracture the subchondral bone plate, consisting of both ACC and underlying SCB. Cracks through the ACC would permit the passage of extracellular fluid, blood or plasma from the underlying bone marrow domain. Without the presence of specific mineralisation inhibitors, whatever the nature of the fluid matrix, it would calcify.

The mineralised cracks we consider here must be distinguished from tissue processing cracks that develop post mortem. The significance of the latter, which are a relatively common feature in the SEM study of PMMA embedded ACC and SCB, has been previously discussed (Boyde, 2003). Cracks may arise during dehydration of the tissue in ethanol prior to substitution with methacrylate monomer, when they will be filled with PMMA after embedding. They may also arise on exposure of the polished block surface to the SEM vacuum due to the removal of firmly bound structural water, which was not substituted with ethanol. Such cracks may enlarge preexisting ante-mortem fissures, but the latter can be reliably distinguished when they have been previously filled with the intercalated highly mineralised matrix.

As we identified mineralised fragments within fibrillated superficial cartilage overlying the projections we surmise that the mineralised matrix can break up to contribute to a population of isolated hard, dense and sharp edged fragments within the HAC. Longitudinal wear lines in the superficial zone of the cartilage are a common 
finding in this joint (Firth et al., 2004; Barr et al., 2009). We have previously proposed that sharp edged ACC and SCB fragments are necessary to account for the formation of wear lines (Boyde and Firth, 2008). Formation of the mineralised extrusions, which we describe in this paper, would constitute another mechanism for introducing hard mineralised material into HAC and eventually into the joint space. However, this is certainly not the only mechanism by which hard and sharp edged osteochondral fragments can arise in this joint. It should be noted that we did not identify any relationship between presence of such features and the occurrence of wear lines at the joint surface. However, we only examined a small proportion of the whole joint's articular surface, and in cases where there were wear lines, there may have been such features in regions we did not study.

The mechanical properties of these dense projections more closely resemble those of tooth enamel than calcified bone or cartilage (Oyen et al., 2008). Nano-indentation identified the matrix of the projections to be stiffer than any normal tissue within the osteochondral organ. On this basis, the material would be predicted to fracture in brittle mode to produce sharp edged shards. Furthermore, the presence of a stiff focus within the HAC would function as a stress riser, predisposing to mechanical disruption of the overlying cartilage matrix. In addition, this could lead to alteration in chondrocyte phenotype to a more degradative state in the region of the projections, due to altered mechanical loading of the cells and surrounding matrix. Such biochemical alterations would lead to matrix loss and ultimately cartilage fibrillation and fibrocartilage formation.

There has been continuing controversy relating to the relative contribution of cartilage and bone pathologies to the OA process (Burr and Radin, 2003; Brandt et al., 2009). Whilst it is likely that both tissues contribute to the disease in many instances, it appears likely that in this form of traumatic OA the primary process begins in the ACC and SCB through formation of traumatic fissures and the cartilage fibrillation is secondary, and subsequent to this process. Whilst it is possible that the ACB and SCB fissuring occurs secondary to cartilage fibrillation, we consider this unlikely as crack formation is observed in such specimens without cartilage degeneration, where there is no extrusion of mineralised material (Boyde and Firth, 2008). Interestingly, we showed no relationship between the presence of such features and a number of measures which correlate with athletic training intensity. The only relationship identified with such features were those related to measures of joint OA, including pathological presence of gross cartilage erosion and increased modified Mankin Score (McIlwraith et al., 2010).

It is of interest that 'central osteophytes' have been described as a pathological feature in both human and equine $\mathrm{OA}$, but it is unclear how these osteophytes relate to our discovery (McCauley et al., 2001; Olive et al., 2009). The examples that we figure in this report would be too small to be detected by clinical imaging means. Indeed, in many cases we had to struggle to find them in Faxitron digital microradiographic imaging of thin sections.

In future work, it would be sensible to re-examine tissues from a variety of human arthropathies, as well as common animal models of OA to determine the presence and the role of such features in a wide range of pathologies. The presence of calcified micro-cracks in ACC in human joint pathology was reported in a microradiographic study by Green et al. (1970). The last author in that study maintained his interest in this observation, and concluded that such in vivo micro-cracks present as basophilic radial hairlines in decalcified tissue histology of ACC (Sokoloff, 1993). He found that they were present in 3 of 25 normal patellae below 50 years, in 20 of 25 recently fractured hips (average age 77 years), and in 16 of 25 osteoarthritic tibial plateaux (72 years). He hypothesised that deep extension of these microfractures might mediate remodelling of the osteochondral junction in ageing and degenerative joint disease. However, extension of the cracks outwards into HAC and an infilling with dense material has not yet been reported in man.

\section{Acknowledgements}

This study was funded by a project grant from the Horserace Betting Levy Board. We are also grateful to the HKJC for financial support and to colleagues within their Department of Veterinary Clinical Services HKJC for access to clinical cases and their assistance with retrieval of material at post mortem. We would like to thank Arthritis Research UK for funding equipment for the point source digital radiography. Mo Arora provided technical assistance throughout this study.

\section{References}

Barr ED, Pinchbeck GL, Clegg PD, Boyde A, Riggs CM (2009) Post mortem evaluation of palmar osteochondral disease (traumatic osteochondrosis) of the metacarpo/ metatarsophalangeal joint in Thoroughbred racehorses. Equine Vet J 41: 366-371.

Boyde A (2003) The real response of bone to exercise. J Anat 203: 173-189.

Boyde A, Firth EC (2008) High resolution microscopic survey of third metacarpal articular calcified cartilage and subchondral bone in the juvenile horse: possible implications in chondro-osseous disease. Microsc Res Tech 71: 477-488.

Boyde A, Haroon Y, Jones SJ, Riggs CM (1999) Three dimensional structure of the distal condyles of the third metacarpal bone of the horse. Equine Vet J 31: 122-129.

Boyde A, Lovicar L, Zamecnik J (2005) Combining confocal and BSE SEM imaging for bone block surfaces. Eur Cell Mater 9: 33-38.

Brandt KD, Dieppe P, Radin E (2009) Etiopathogenesis of osteoarthritis. Med Clin North Am 93: 1-24, xv.

Burr DB, Radin EL (2003) Microfractures and microcracks in subchondral bone: are they relevant to osteoarthrosis? Rheum Dis Clin North Am 29: 675-685.

Eidelman N, Boyde A, Bushby AJ, Howell PGT, Sun J, Newbury DE, Miller FW, Robey PG, Rider LG. (2009) Microstructure and mineral composition of dystrophic 
calcification associated with the idiopathic inflammatory myopathies. Arthritis Res Ther 11: R159.

Ferguson VL, Bushby AJ, Boyde A (2003) Nanomechanical properties and mineral concentration in articular calcified cartilage and subchondral bone. J Anat 203: 191-202.

Firth EC, Rogers CW, Perkins NR, Anderson BH (2004) Musculoskeletal responses of two year old thoroughbred horses to early training. 1. Study design, and clinical, nutritional, radiological, and histological observations. NZ Vet J 52: 261-271.

Firth EC, Doube M, Boyde A (2009) Changes in mineralised tissue at the site of origin of condylar fracture are present before athletic training in Thoroughbred horses. N Z Vet J 57: 278-283.

Green WT, Martin GN, Eanes ED, Sokoloff L (1970) Microradiographic study of the calcified layer or articular cartilage. Arch Pathol 90: 51-158.

McCauley TR, Kornaat PR, Jee WH (2001) Central osteophytes in the knee: prevalence and association with cartilage defects on MR imaging. Am J Roentgenol 176: 359-364.

McIlwraith CW, Frisbie DD, Kawcak CE, Fuller CJ, Hurtig M, Cruz A (2010) The OARSI histopathology initiative - recommendations for histological assessments of osteoarthritis in the horse. Osteoarthritis Cartilage, 18 Suppl 3: S93-105.

Norrdin RW, Stover SM (2006) Subchondral bone failure in overload arthrosis: a scanning electron microscopic study in horses. J Musculoskelet Neuronal Interact 6: 251-257.

Olive J, D'Anjou MA, Girard C, Laverty S, Theoret CL (2009) Imaging and histological features of central subchondral osteophytes in racehorses with metacarpophalangeal joint osteoarthritis. Equine Vet J 41: 859-864.

Oyen ML, Ferguson VL, Bembey AK, Bushby AJ, Boyde A (2008) Composite bounds on the elastic modulus of bone. J Biomech 41: 2585-2588.

Sokoloff L (1993) Micro-cracks in the calcified layer of articular cartilage. Arch Pathol Lab Med 117: 191-195.

\section{Discussion with Reviewers}

Reviewer I: Can you comment on how this pathology might relate to the process of micro-fracturing as a technique for articular cartilage repair?

Authors: This technique, which involves punching small holes through an articular surface that has lost all soft cartilage. What we describe involves changes within some intact HAC.

Reviewer II: Were the horses killed because of welfare causes directly related to the joints that were examined? Authors: All the horses were destroyed because they had either sustained an acute, severe injury for which there was no reasonable treatment or because they were suffering from a chronic condition, usually musculoskeletal in origin, which was progressive in nature and judged to affect the horse's quality of life in retirement. A proportion of these cases included conditions that directly affected the joint(s) from which material used in the study was collected.

Reviewer II: The authors mention that the subchondral plate $=\mathrm{ACC}+\mathrm{SCB}$. Is this a new definition? If it is not, please cite the original reference. If it is, please support more robustly so that ACC is more likely to be recognised as a tissue of significance.

Authors: All clinical imaging modalities and standard laboratory micro CT equipment provide images in which it is impossible to distinguish ACC from SCB. The reason that we mention that the subchondral plate is made of both tissues is that it is so. Of course, by this definition it is strictly a sub-HAC plate. Using decalcified section histology, the ACC can be distinguished, but then its mineral content has gone, and the inherently interesting detail concerning micron level variations in mineralisation has been thrown away with the decalcifying solution.

It is almost as if ACC did not exist as a separate tissue entity in much of the clinical research world. Take for example a very recent paper e.g., Aula et al. (2010), but there are hundreds of others. BSE SEM imaging currently has a unique advantage over all other methods in providing the detail necessary to study ACC, but it lacks input concerning non-calcified elements, including cells and matrix. This is the reason why we introduced the method of correlation of identical image fields by BSE SEM and CSLM (Boyde et al., 2005, text reference) and which we put to good use in making the present discovery.

Reviewer II: Please could you propose how you would show that the changes that you have so beautifully shown are indeed a mechanism of early articular cartilage pathology?

Authors: It is quite improbable that we can 'prove' this because it would require the inspection of all cartilage in affected joints at the time of euthanasia to search for fragments of mineralised tissue embedded in HAC or loose in the joint fluid, in a large number of animals. We consider ourselves fortunate to have found the few cases that we did in a large number of animals. Making others aware of the existence of the calcified extrusions should encourage them to search for traces in their own studies.

\section{Additional References}

Aula AS, Töyräs J, Tiitu V, Jurvelin JS (2010) Simultaneous ultrasound measurement of articular cartilage and subchondral bone. Osteoarthritis Cartilage 18: $1570-1576$. 